\title{
EXTREME ANALYSIS OF A RANDOM ORDINARY DIFFERENTIAL EQUATION
}

\author{
JINGCHEN LIU,* Columbia University \\ XIANG ZHOU,** City University of Hong Kong
}

\begin{abstract}
In this paper we consider a one dimensional stochastic system described by an elliptic equation. A spatially varying random coefficient is introduced to account for uncertainty or imprecise measurements. We model the logarithm of this coefficient by a Gaussian process and provide asymptotic approximations of the tail probabilities of the derivative of the solution.

Keywords: Random differential equation; extremes; rare event

2010 Mathematics Subject Classification: Primary 60F10

Secondary $65 Z 05$
\end{abstract}

\section{Introduction}

In this paper we consider the tail event that arises naturally from a differential equation when employed in various applications. Very often microscopic heterogeneity or uncertainty in the parameters exist such that the system cannot be completely characterized by a deterministic differential equation. Stochastic models are usually employed, in combination with differential equations, to account for such heterogeneity and/or uncertainty. In this paper we are interested in one specific differential equation concerning a real-valued solution $v(x)$

$$
\left(a(x) v^{\prime}(x)\right)^{\prime}=p(x), \quad x \in[0, L],
$$

where $a(x)$ and $p(x)$ are real-valued functions. This equation has applications to several subfields of physics and also has a close connection to stochastic differential equations.

In this paper we adopt the formulation that the process $a(x)$ is a spatially varying stochastic process and thus the corresponding solution $v(x)$ is itself (as a function of $a(x)$ ) also a stochastic process. In a physical model the process $a(x)$ is constrained to be positive. A natural modeling approach is to accept that $a(x)$ is a log-normal process,

$$
a(x)=\mathrm{e}^{-\sigma \xi(x)}, \quad \sigma>0,
$$

where $\xi(x)$ is a Gaussian process living on $[0, L]$. We are interested in developing sharp asymptotic approximations of the tail probabilities associated with $v(x)$, in particular,

$$
w(b) \stackrel{\mathrm{D}}{=} \mathbb{P}\left(\max _{x}\left|v^{\prime}(x)\right|>b\right) \quad \text { as } b \rightarrow \infty .
$$

Such tail probabilities serve as a risk measure for the material failure of an elastic body under the maximum strain (i.e. $\left.v^{\prime}(x)\right)$ criteria [10].

Received 5 July 2013; revision received 13 November 2013.

* Postal address: Department of Statistics, Columbia University, 1255 Amsterdam Avenue, New York, NY 10027, USA. Email address: jcliu@ @stat.columbia.edu

** Postal address: Y6524 (Yellow Zone), 6/F Academic 1, City University of Hong Kong, Tat Chee Avenue, Kowloon Tong, Hong Kong. 
Under the Dirichlet boundary condition, $u(0)=u(L)=0$, and with representation (2), equation (1) has a closed form solution

$$
v(x)=\int_{0}^{x} F(t) \mathrm{e}^{\sigma \xi(t)} \mathrm{d} t-\int_{0}^{x} \mathrm{e}^{\sigma \xi(t)} \mathrm{d} t \int_{0}^{L} F(s) \mathrm{e}^{\sigma \xi(s)} \mathrm{d} s / \int_{0}^{L} \mathrm{e}^{\sigma \xi(s)} \mathrm{d} s,
$$

where $F(x) \stackrel{\mathrm{D}}{=} \int_{0}^{x} p(t) \mathrm{d} t$ and its derivative is

$$
v^{\prime}(x)=\mathrm{e}^{\sigma \xi(x)}\left\{F(x)-\frac{\int_{0}^{L} F(t) \mathrm{e}^{\sigma \xi(t)} \mathrm{d} t}{\int_{0}^{L} \mathrm{e}^{\sigma \xi(t)} \mathrm{d} t}\right\} .
$$

In this paper we present the derivation of closed form sharp asymptotic approximations of $w(b)$ as $b \rightarrow \infty$. In particular, we discuss two situations: (i) $p(x)$ is a constant and (ii) $|p(x)|$ admits one unique maximum in the interior of $[0, L]$. In addition to the asymptotic approximations of $w(b)$ this analysis also implies qualitative descriptions of the most likely sample path along which $\max _{x}\left|v^{\prime}(x)\right|$ achieves a high level. Firstly, if $p(x)$ is a constant then the maximum of $\left|v^{\prime}(x)\right|$ is likely to be obtained at either end of the interval and is unlikely to be obtained in the interior. Secondly, if $|p(x)|$ admits one unique interior maximum at $x_{*}=\arg \max _{x}|p(x)|$, then the maximum of $\left|v^{\prime}(x)\right|$ is likely to be obtained at either of the three locations, $0, L$, or close to $x_{*}$, depending on the specific values of $p(0), p(L)$, and $p\left(x_{*}\right)$.

By considering max $\left|v^{\prime}(x)\right|$ as a functional of the input Gaussian process $\xi(x)$ the analysis presented here is consistent with the published literature concerning rare-event analysis for Gaussian processes (see, for example, [1]-[9], [11]-[14]). The analysis combines an understanding of physics, which helps with guessing the most probable sample path of $\xi(x)$ given the high excursion of $\left|v^{\prime}(x)\right|$, and random field techniques to derive approximations of $w(b)$.

The rest of this paper is organized as follows. In Section 2 we present the main results and in Section 3 we prove the theorems. Supplementary material is provided at [15], while a more comprehensive manuscript is available at [16] containing more discussions on the applications of our results.

\section{Main results}

We consider the differential equation (1) that has the Dirichlet condition. The gradient of the solution of (1) is given by (3). The random coefficient $a(x)$ takes the form of (2), where $\xi(x)$ is a Gaussian process living on $[0, L]$. Next we list a set of technical conditions concerning the input process $\xi(x)$ and the function $p(x)$.

Assumption 1. The process $\xi(x)$ is strongly stationary with $\mathbb{E}[\xi(x)]=0$ and $\mathbb{E}\left[\xi^{2}(x)\right]=1$.

Assumption 2. The process $\xi(x)$ is almost surely three-time differentiable. The covariance function admits the following expansion $\operatorname{cov}(\xi(0), \xi(x))=C(x)=1-\frac{1}{2} \Delta x^{2}+\frac{1}{24} A x^{4}-B x^{6}$ $+o\left(x^{6}\right)$, as $x \rightarrow 0$. In addition, for each $x, C(\lambda x)$ is a nonincreasing function of $\lambda \in \mathbb{R}^{+}$.

Assumption 3. The function $p(x)$ is at least twice continuously differentiable. In addition, it falls into either of the two cases.

Case 1. $|p(x)|$ admits its unique interior global maximum $x_{*}=\arg \max |p(x)|$ and $x_{*} \in$ $(0, L)$. Furthermore, $|p(x)|$ is strongly concave (meaning that the second derivative is strictly negative) in a sufficiently small neighborhood around $x_{*}$.

Case 2. $p(x)$ is constant. 
Assumption 2 is an important assumption for the entire analysis. In particular, three-time differentiability implies that the covariance function is at least six-time differentiable and that the first, third, and fifth derivatives when evaluated at the origin are all zero. The coefficients $\Delta$ and $A$ are known as the spectral moments, and these are discussed further in the later analysis. In Assumption 3, if $|p(x)|$ has more than one (interior) global maximum or the global maximum is at the boundary then the analysis can be adapted.

In the following we first consider Assumption 3, Case 1 where $|p(x)|$ admits one unique maximum. Let $x_{*} \stackrel{D}{=} \arg \max _{x \in[0, L]}|p(x)|$ be the unique interior maximum in $(0, L)$. Without loss of generality we assume that $p\left(x_{*}\right), p(0)$, and $p(L)$ are all positive. For the case that some, or all, of them are negative then the analysis is completely analogous. This will be mentioned in later remarks.

We define three variables $u, u_{0}$ and $u_{L}$ that depend on the excursion level $b$. They are all approximately on the scale of $(\log b) / \sigma$. For each $b>0$, let $u$ be the solution to the nonlinear equation

$$
p\left(x_{*}\right) H\left(\gamma_{*}(u), u\right) \mathrm{e}^{\sigma u}=b,
$$

where

$$
H(x, u) \triangleq|x| \mathrm{e}^{-\Delta \sigma u x^{2} / 2},
$$

and $\gamma_{*}(u) \stackrel{\mathrm{D}}{=} \arg \sup _{x>0} H(x, u)=1 /(\sqrt{u \Delta \sigma})$. Identity (4) can be further simplified to give $\left.\left(p\left(x_{*}\right) / \sqrt{\sigma \Delta u}\right) \exp (1 / \sqrt{\sigma u})\right)=b$. We introduce the notation $\gamma_{*}(u)$ and $H$ because they arise naturally in the derivation and they have geometric and probabilistic interpretations that will be given in the proof of our main theorems.

For each $b>0$, let $u_{0}$ be the solution to

$$
\frac{\mathrm{e}^{\sigma u_{0}}}{\sqrt{\Delta \sigma u_{0}}} \sup _{\{(x, \zeta): x \leq \zeta\}} H_{0}\left(x, \zeta ; u_{0}\right)=b
$$

where

$$
H_{0}(x, \zeta ; u) \stackrel{\mathrm{D}}{=} \mathrm{e}^{-x^{2} / 2} \mathbb{E}\left[p(0)(x-Z)+\frac{p^{\prime}(0)}{2 \sqrt{\Delta \sigma u}}(x-Z)^{2} \mid Z \leq \zeta\right]
$$

$Z$ is a standard Gaussian random variable independent of any other randomness in the system and where $\mathbb{E}[\cdot \mid Z \leq \zeta]$ denotes the conditional expectation with respect to $Z$ given by $Z \leq \zeta$. We provide further explanations of $H_{0}$. The second term inside the expectation (6) is $o(1)$ and thus $H_{0}(x, \zeta ; u) \approx p(0) \exp \left(-x^{2} / 2\right)(x-\mathbb{E}[Z \mid Z \leq \zeta])$. The last term in the definition of $H_{0}$ is important in order to obtain a sharp approximation of the tail probabilities. More properties of $H_{0}$ are included in Remark 1. Similarly, we define $u_{L}$ by

$$
\frac{\mathrm{e}^{\sigma u_{L}}}{\sqrt{\Delta \sigma u_{L}}} \sup _{\{(x, \zeta): x \leq \zeta\}} H_{L}\left(x, \zeta ; u_{L}\right)=b
$$

where $H_{L}(x, \zeta ; u)$ is defined in a similar way as in (6) except replacing $p(0)$ and $p^{\prime}(0)$ by $p(L)$ and $-p^{\prime}(L)$, respectively.

Function $F(x)$ is bounded and the factor, $F(x)-\int_{0}^{L} F(t) \exp (\xi(t)) \mathrm{d} t / \int_{0}^{L} \exp (\xi(t)) \mathrm{d} t$, is also bounded. In fact, this factor converges to zero under the conditional distribution given the high excursion of $\left|v^{\prime}(x)\right|$. Thus, if $\left|v^{\prime}(x)\right|$ exhibits a high excursion then $\xi(x)$ must also achieve a high excursion level. The variable $u$ is interpreted as the level that $\xi(x)$ needs to achieve so that $\left|v^{\prime}(x)\right|$ achieves the level $b$ around $x_{*}$. Similarly, $u_{0}$ and $u_{L}$ correspond to the high excursion levels of $\xi(x)$ at the two ends. 
For each $\zeta, u_{0}$, and $u_{L}$, maximizing $\log \left(\left|H_{0}\right|\right)$ and $\log \left(\left|H_{L}\right|\right)$ over $x \in(-\infty, \zeta]$ gives us the definitions of the following functions:

$$
G_{0}\left(\zeta ; u_{0}\right) \stackrel{\mathrm{D}}{=} \sup _{x \leq \zeta} \log \left|H_{0}\left(x, \zeta ; u_{0}\right)\right| \quad \text { and } \quad G_{L}\left(\zeta ; u_{L}\right) \stackrel{\mathrm{D}}{=} \sup _{x \leq \zeta} \log \left|H_{L}\left(x, \zeta ; u_{L}\right)\right| .
$$

Define the maximizers of the $G$-function as

$$
\zeta_{0} \stackrel{\mathrm{D}}{=} \arg \max _{\zeta} G_{0}\left(\zeta ; u_{0}\right) \quad \text { and } \quad \zeta_{L} \stackrel{\mathrm{D}}{=} \arg \max _{\zeta} G_{L}\left(\zeta ; u_{L}\right)
$$

Note that $\zeta_{0}$ depends on $u_{0}$ and $\zeta_{L}$ depends on $u_{L}$. To simplify the notation we omit the indices $u_{0}$ and $u_{L}$ in the notation $\zeta_{0}$ and $\zeta_{L}$ when there is no ambiguity. The second derivatives of the $G$-functions, evaluated at their maximizers, are $\Xi_{0} \stackrel{\mathrm{D}}{=}-\left.\lim _{u_{0} \rightarrow \infty} \partial_{\zeta}^{2} G_{0}\right|_{\zeta=\zeta_{0}, u=u_{0}}$, and $\Xi_{L} \stackrel{\mathrm{D}}{=}-\left.\lim _{u_{L} \rightarrow \infty} \partial_{\zeta}^{2} G_{L}\right|_{\zeta=\zeta_{L}, u=u_{L}}$, respectively. Finally, we define the constant

$$
\begin{aligned}
\kappa_{0} \stackrel{\mathrm{D}}{=} & \frac{A \zeta_{0}}{24 \Delta^{2} \sigma}-\frac{A \times \mathbb{E}\left[Z^{4} \mid Z \leq \zeta_{0}\right]}{24 \Delta^{2} \sigma} \\
& +\frac{\mathbb{E}\left[\left(p^{\prime \prime}(0) /(6 \sigma \Delta)\right)\left(\zeta_{0}-Z\right)^{3}+\left(A p(0) /\left(24 \Delta^{2} \sigma^{2}\right)\right) Z^{4}\left(\zeta_{0}-Z\right) \mid Z \leq \zeta_{0}\right]}{p(0) \mathbb{E}\left[\zeta_{0}-Z \mid Z \leq \zeta_{0}\right]}
\end{aligned}
$$

as well as $\kappa_{L}$, which is similar to the above, by replacing $\zeta_{0}$ with $\zeta_{L}$. The main results are summarized in the following theorems.

Theorem 1. Suppose that $\xi(x)$ is a Gaussian process satisfying Assumptions 1 and 2 and Case 1 of Assumption 3. For all $x \in[0, L]$, let $v^{\prime}(x)$ be given as in (3). Let $u, u_{0}$, and $u_{L}$ be defined as above. If $p(x)$ is nonnegative at $x=0, x_{*}$, and $L$, then $\mathbb{P}\left(\sup _{x \in[0, L]}\left|v^{\prime}(x)\right|>\right.$ $b) \sim(D / \sqrt{u}) \exp \left(-u^{2} / 2\right)+\left(D_{0} / u_{0}\right) \exp \left(-u_{0}^{2} / 2\right)+\left(D_{L} / u_{L}\right) \exp \left(-u_{L}^{2} / 2\right)$, where $D, D_{0}$, and $D_{L}$ are constants defined as

$$
\begin{aligned}
D= & \frac{\sqrt{\Delta} \exp \left[\left(A /\left(24 \sigma^{2} \Delta^{2}\right)\right)+\left(p^{\prime \prime}\left(x_{*}\right) /\left(6 p\left(x_{*}\right) \sigma^{2} \Delta\right)\right]\right.}{(2 \pi)^{3 / 2} \sqrt{A-\Delta^{2}}} \\
& \times \int \exp \left\{-\frac{1}{2}\left[\frac{\Delta^{2} z^{2}}{A-\Delta^{2}}-\frac{z}{\sigma}-\frac{y^{2} z}{\Delta}+\frac{A y^{4}}{4 \Delta^{4}}+\frac{A y^{2}}{2 \sigma \Delta^{3}}-\frac{p^{\prime \prime}\left(x_{*}\right) y^{2}}{p\left(x_{*}\right) \sigma \Delta^{2}}\right]\right\} \mathrm{d} y \mathrm{~d} z, \\
D_{0}= & \frac{\sqrt{\Delta} \exp \left(\kappa_{0} / \sigma\right)}{(2 \pi)^{3 / 2} \sqrt{A-\Delta^{2}}} \int \exp \left(-\frac{1}{2}\left(\frac{\Delta^{2} z^{2}}{A-\Delta^{2}}-\frac{z}{\sigma}+\frac{\Xi_{0}}{\Delta} y^{2}\right)\right) \mathrm{d} y \mathrm{~d} z, \\
D_{L}= & \frac{\sqrt{\Delta} \exp \left(\kappa_{L} / \sigma\right)}{(2 \pi)^{3 / 2} \sqrt{A-\Delta^{2}}} \int \exp \left(-\frac{1}{2}\left(\frac{\Delta^{2} z^{2}}{A-\Delta^{2}}-\frac{z}{\sigma}+\frac{\Xi_{L}}{\Delta} y^{2}\right)\right) \mathrm{d} y \mathrm{~d} z .
\end{aligned}
$$

If $p(x)$ attains its maximum at multiple interior points $x_{1}, \ldots, x_{k}$, then the approximation becomes $\mathbb{P}\left(\sup _{x \in[0, L]}\left|v^{\prime}(x)\right|>b\right) \sim \sum_{j=1}^{k} D(j)(1 / \sqrt{u}) \exp \left(-u^{2} / 2\right)+D_{0} u_{0}^{-1} \exp \left(-u_{0}^{2} / 2\right)+$ $\left(D_{L} / u_{L}\right) \exp \left(-u_{L}^{2} / 2\right)$, where $D(j)$ 's are defined similarly as $D$ except by replacing $x_{*}$ with $x_{k}$. If the maximizer $x_{*}$ is attained on the boundary, then the term $(D / \sqrt{u}) \exp \left(-u^{2} / 2\right)$ should be removed from the approximation.

The theorem assumes that $p(x)$ is positive at the important locations. In the case when $p\left(x_{*}\right)<0$, we simply define $u$ through $\left|p\left(x_{*}\right)\right| \exp \left(\sigma u+H\left(\gamma_{*}(u), u\right)\right)=b$. The definitions of other variables remain. Similarly, if $p(0)$ is negative we should generally define that

$$
H_{0}(x, \zeta ; u) \stackrel{\mathrm{D}}{=} \operatorname{sgn}(p(0)) \mathrm{e}^{-x^{2} / 2} \times \mathbb{E}\left[p(0)(x-Z)+\frac{p^{\prime}(0)}{2 \sqrt{\Delta \sigma u}}(x-Z)^{2} \mid Z \leq \zeta\right]
$$




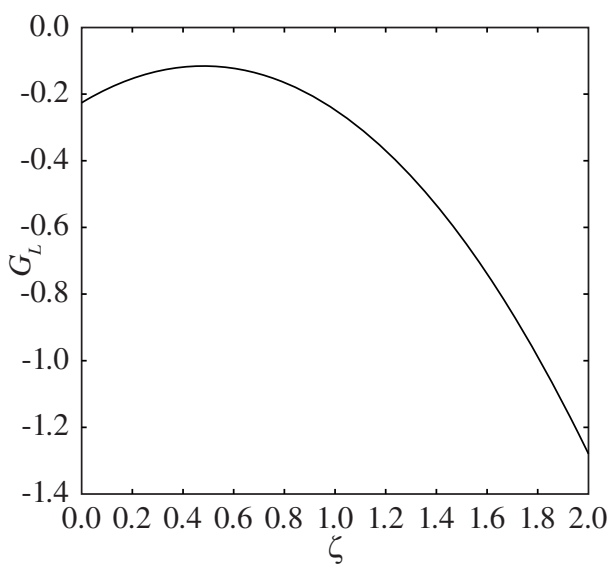

FIGURE 1: Function $G_{L}\left(\zeta, u_{L}=\infty\right)$.

where 'sgn' is the sign function. The same treatment can be applied to $H_{L}$ when $p(L)$ is negative. The rest of the definitions remain. To simplify the notation we assume that $p(0)$ and $p(L)$ are positive and do not include the sgn term.

Remark 1. There are several features of the functions $H_{0}$ and $H_{L}$ that are important in the analysis. As $u_{L} \rightarrow \infty$, we have that $H_{L}\left(x, \zeta ; u_{L}\right) \rightarrow p(L) \exp \left(-x^{2} / 2\right)(x-\mathbb{E}[Z \mid Z \leq \zeta])>$ 0 and $\zeta_{L} \approx 0.48$. In addition, for $\zeta \leq 0.84$, we have $\left.\left(\left(\partial\left|H_{L}\right|\right) /(\partial x)\right)\right|_{(x, \zeta)=(\zeta, \zeta)}>0$, and thus $\max _{x \in(-\infty, \zeta]} \log \left|H_{L}(x, \zeta)\right|$ is solved at $x=\zeta$, that is, $G_{L}\left(\zeta ; u_{L}\right)=\log \left|H_{L}\left(\zeta, \zeta ; u_{L}\right)\right|$. This calculation is important in the technical derivations and it ensures that the maximum of $\left|v^{\prime}(x)\right|$ is attained precisely at $x=L$ if $\max _{L-\varepsilon<x \leq L}\left|v^{\prime}(x)\right|>b$. To assist understanding we numerically compute the function $G_{L}$ for $\zeta>0$ by setting $u_{L}=\infty$. This is shown in Figure 1 for $p(L)=1$.

Now we proceed to the approximation of $w(b)$ when $p(x) \equiv p_{0}>0$. The approximation is very similar to Theorem 1 , except that we do not have the term $D \times(1 / \sqrt{u}) \exp \left(-u^{2} / 2\right)$ and all the derivatives of $p(x)$ vanish. To state the theorem we need the following notation. We define a similar $H$-function and $G$-function as $H_{h}(x, \zeta)=p_{0} \exp \left(-x^{2} / 2\right) \mathbb{E}[x-Z \mid Z \leq \zeta]$, and $G_{h}(\zeta)=\sup _{x \leq \zeta} \log \left|H_{h}(x, \zeta)\right|$. Furthermore, we define constants $\zeta_{h}=\arg \sup _{\zeta} G_{h}(\zeta)$, $\Xi_{h}=-\partial_{\zeta}^{2} G_{h}\left(\zeta_{h}\right)$

$$
\begin{aligned}
D_{h} & =\frac{\Delta \exp \left(\kappa_{h} / \sigma\right)}{(2 \pi)^{3 / 2} \sqrt{A-\Delta^{2}}} \int \exp \left\{-\frac{1}{2}\left[\frac{\Delta^{2} z^{2}}{A-\Delta^{2}}-\frac{z}{\sigma}+\frac{\Xi_{h}}{\Delta} y^{2}\right]\right\} \mathrm{d} y \mathrm{~d} z, \\
\kappa_{h} & =\frac{A \zeta_{h}^{4}}{24 \Delta^{2} \sigma}-\frac{A \mathbb{E}\left[Z^{4} \mid Z \leq \zeta_{h}\right]}{24 \Delta^{2} \sigma}+\frac{A \mathbb{E}\left[Z^{4}\left(\zeta_{h}-Z\right) \mid Z \leq \zeta_{h}\right]}{24 \Delta^{2} \sigma^{2} \mathbb{E}\left[\zeta_{h}-Z \mid Z \leq \zeta_{h}\right]} .
\end{aligned}
$$

Theorem 2. Suppose that the random field $\xi(x)$ satisfies the Assumptions 1 and 2 and Case 2 of Assumption 3. In addition, the external force $p(x) \equiv p_{0}$ is a positive constant. For each $b>0$, let $u_{h}$

$$
\frac{\exp \left(\sigma u_{h}\right)}{\sqrt{\Delta \sigma u_{h}}} \sup _{\{(x, \zeta): x \leq \zeta\}} H_{h}(x, \zeta)=b .
$$

Then, we have the closed form approximation

$$
\mathbb{P}\left(\sup _{x \in[0, L]}\left|v^{\prime}(x)\right|>b\right) \sim 2\left(D_{h} / u_{h}\right) \exp \left(-u_{h}^{2} / 2\right) .
$$


The proof of Theorem 2 is very similar to that of Theorem 1 . We present it in the supplementary material [15], [16]. We further provide intuitive interpretations of the previous asymptotic approximations. In particular, we focus mostly on the case when $p(x)$ is not a constant.

The approximation in Theorem 1 consists of three terms. The first term $(D / \sqrt{u}) \exp \left(-u^{2} / 2\right)$ corresponds to the probability that the maximum of $\left|v^{\prime}(x)\right|$ is attained close to the interior point $x_{*}=\arg \max _{x \in[0, L]}|p(x)|$ where the terms $\left(D_{0} / u_{0}\right) \exp \left(-u_{0}^{2} / 2\right)$ and $\left(D_{L} / u_{L}\right) \exp \left(-u_{L}^{2} / 2\right)$ correspond to the probabilities that the excursion of $\left|v^{\prime}(x)\right|$ occurs at the two boundary points $x=0$ and $x=L$, respectively. Thus, this three-term decomposition of $w(b)$ suggests that the conditional probability

$$
\mathbb{P}\left(\max _{x \in\left[\varepsilon, x_{*}-\varepsilon\right] \cup\left[x_{*}+\varepsilon, L-\varepsilon\right]}\left|v^{\prime}(x)\right|>b\left|\max _{[0, L]}\right| v^{\prime}(x) \mid>b\right) \rightarrow 0,
$$

as $b \rightarrow \infty$ for any $\varepsilon>0$. It is unlikely that the maximum is attained at any location other than the two ends or at $x_{*}$. As for which of the three locations is most likely to exhibit a high excursion, it depends on the specific functional forms of $p(x)$. Note that all the three terms decay exponentially fast with $u^{2}, u_{0}^{2}$, or $u_{L}^{2}$. Therefore, the smallest value among $u, u_{0}$, and $u_{L}$ corresponds to the most likely location. Note that $u_{0}$ and $u_{L}$ take the same form. Thus, we only need to compare $|p(0)|$ and $|p(L)|$. The larger one corresponds to a smaller $u$-value and therefore yields a more likely high excursion. To compare the boundary case and the interior case we need to compare $u$ and $u_{0}$ (or $u_{L}$ ). We take $u_{0}$ as an example. Note that both $u$ and $u_{0}$ are defined by $b$ implicitly through the equations having similar forms. Therefore, it is sufficient to compare among the two terms

$$
\left|p\left(x_{*}\right) \mathrm{e}^{H\left(\gamma_{*}, u\right)}\right|=\left|p\left(x_{*}\right)\right| \frac{\mathrm{e}^{-1 / 2}}{\sqrt{\sigma \Delta u}},
$$

and

$$
\frac{\sup _{x \leq \zeta} H_{0}\left(x, \zeta, u_{0}\right)}{\sqrt{\sigma \Delta u_{0}}} \sim \frac{|p(0)| \sup _{x \leq \zeta} \mathrm{e}^{-x^{2} / 2} \mathbb{E}[x-Z \mid Z \leq \zeta]}{\sqrt{\sigma \Delta u}} .
$$

Furthermore, we consider the ratio

$$
r \stackrel{\mathrm{D}}{=} \frac{\sup _{x \leq \zeta} \mathrm{e}^{-x^{2} / 2} \mathbb{E}[x-Z \mid Z \leq \zeta]}{\sqrt{\sigma \Delta u}} / \frac{\mathrm{e}^{-1 / 2}}{\sqrt{\sigma \Delta u}}=\sup _{(\zeta, x), \text { s.t. } x \leq \zeta} \mathrm{e}^{\left(1-x^{2}\right) / 2} \mathbb{E}[x-Z \mid Z \leq \zeta] .
$$

Note that $r$ is a universal constant strictly greater than 1. If $\left|p\left(x_{*}\right)\right|>r|p(0)|$, then $x_{*}$ is a more probable location to observe a high excursion but if $\left|p\left(x_{*}\right)\right|<r|p(0)|$, then zero is a more probable location. If $p(x)$ is a constant, then $u>u_{0}=u_{h}$. This is why the maximum of $v^{\prime}(x)$ is not attained in the interior for this case.

\section{Proof of Theorem 1}

In order not to overcomplicate the discussion, we present the proof of all supporting propositions and lemmas in the supplementary material, see [15], [16]. The proof in Theorem 1 is based on the following inclusion-exclusion equation

$$
\sum_{i=1}^{3} \mathbb{P}\left(\mathbb{E}_{i}\right)-\sum_{i=1}^{2} \sum_{j=i+1}^{3} \mathbb{P}\left(\mathbb{E}_{i} \cap \mathbb{E}_{j}\right) \leq \mathbb{P}\left(\max _{[0, L]} v^{\prime}(x)>b\right)=\mathbb{P}\left(\bigcup_{i=1}^{3} \mathbb{E}_{i}\right) \leq \sum_{i=1}^{3} \mathbb{P}\left(\mathbb{E}_{i}\right),
$$


where

$$
\begin{aligned}
& \mathbb{E}_{1}=\left\{\max _{x \in\left[u^{-1 / 2+\delta}, L-u^{-1 / 2+\delta}\right]}\left|v^{\prime}(x)\right|>b\right\}, \\
& \mathbb{E}_{2}=\left\{\max _{x \in\left[0, u^{-1 / 2+\delta}\right]}\left|v^{\prime}(x)\right|>b\right\}, \text { and } \\
& \mathbb{E}_{3}=\left\{\max _{x \in\left[L-u^{-1 / 2+\delta}, L\right]}\left|v^{\prime}(x)\right|>b\right\},
\end{aligned}
$$

for some $\delta>0$ sufficiently small but independent of $b$. The main body is to derive the approximations for $\mathbb{P}\left(\mathbb{E}_{i}\right)$. In addition, from the following detailed derivation of $\mathbb{P}\left(\mathbb{E}_{1}\right)$ and $\mathbb{P}\left(\mathbb{E}_{3}\right)$, it is straightforward to write

$$
\mathbb{P}\left(\mathbb{E}_{1} \cap \mathbb{E}_{2}\right)+\mathbb{P}\left(\mathbb{E}_{1} \cap \mathbb{E}_{3}\right)+\mathbb{P}\left(\mathbb{E}_{2} \cap \mathbb{E}_{3}\right)=o\left(\mathbb{P}\left(\mathbb{E}_{1}\right)+\mathbb{P}\left(\mathbb{E}_{2}\right)+\mathbb{P}\left(\mathbb{E}_{3}\right)\right) .
$$

Thus, we complete the proof of Theorem 1 by the inclusion-exclusion equation. In the following analysis we will use both $x$ and $t$ to denote the spatial index. In particular, we use $t$ for the index when performing integration and use $x$ when taking the supremum.

\subsection{Approximation for $\mathbb{P}\left(\mathbb{E}_{1}\right)$}

Consider the following change of variables from $\left(\xi\left(x_{*}\right), \xi^{\prime}\left(x_{*}\right), \xi^{\prime \prime}\left(x_{*}\right)\right)$ to $(w, y, z)$ that depend on the variable $u, w \stackrel{\mathrm{D}}{=} \xi\left(x_{*}\right)-u, y \stackrel{\mathrm{D}}{=} \xi^{\prime}\left(x_{*}\right)$, and $z \stackrel{\mathrm{D}}{=} u+\xi^{\prime \prime}\left(x_{*}\right) / \Delta$. Additionally we write $\mathbb{P}\left(\cdot \mid \xi\left(x_{*}\right)=u+w, \xi^{\prime}\left(x_{*}\right)=y, \xi^{\prime \prime}\left(x_{*}\right)=-\Delta(u-z)\right)=\mathbb{P}(\cdot \mid w, y, z)$ and obtain

$$
\mathbb{P}\left(\mathbb{E}_{1}\right)=\Delta \int \mathbb{P}\left(\mathbb{E}_{1} \mid w, y, z\right) h(w, y, z) \mathrm{d} w \mathrm{~d} y \mathrm{~d} z,
$$

where $h(w, y, z)$ is the density function of $\left(\xi\left(x_{*}\right), \xi^{\prime}\left(x_{*}\right), \xi^{\prime \prime}\left(x_{*}\right)\right)$ evaluated at $(u+w, y,-\Delta(u$ $-z)$ ). The following proposition localizes the event to a region that is convenient for a Taylor expansion on $\xi(x)$.

Proposition 1. Under the conditions in Theorem 1, consider

$$
\mathcal{L}_{u}=\left\{|w|<u^{3 \delta}\right\} \cap\left\{|y|<u^{1 / 2+4 \delta}\right\} \cap\left\{|z|<u^{1 / 2+4 \delta}\right\}
$$

Then, for any $\delta>0$, we have that $\mathbb{P}\left(\mathcal{L}_{u}^{c} ; \mathbb{E}_{1}\right)=o\left(u^{-1} \mathrm{e}^{-u^{2} / 2}\right)$.

This proposition localizes the event $\mathbb{E}_{1}$ to a region where the maximum of $v^{\prime}(x)$ is achieved around $x_{*}$. The above proposition suggests that we only need to consider the event on the set $\mathcal{L}_{u}$, that is, $\Delta \int_{\mathcal{L}_{u}} \mathbb{P}\left(\mathbb{E}_{1} \mid w, y, z\right) h(w, y, z) \mathrm{d} w \mathrm{~d} y \mathrm{~d} z$.

Conditional on $\left(\xi\left(x_{*}\right), \xi^{\prime}\left(x_{*}\right), \xi^{\prime \prime}\left(x_{*}\right)\right)$, we write the process in the following representation $\xi(x)=\mathbb{E}(\xi(x) \mid w, y, z)+g\left(x-x_{*}\right)$. The process $g\left(x-x_{*}\right)$ represents the variation of $\xi(x)$ when $\xi\left(x_{*}\right)$ and its first two derivatives have been fixed. Thus, $g\left(x-x_{*}\right)$ is a mean-zero Gaussian process almost surely three-time differentiable. Using conditional Gaussian calculations and a Taylor expansion we have that $\operatorname{var}\left(g\left(x-x_{*}\right)\right)=O\left(\left|x-x_{*}\right|^{6}\right)$, that is, $g\left(x-x_{*}\right)=O_{p}\left(\left|x-x_{*}\right|^{3}\right)$ as $g$ is the remainder term after conditioning on $\xi\left(x_{*}\right)$ and the first two derivatives. Note that the distribution of $g(x)$ is free of $(w, y, z)$. Let $\mathbb{E}(x ; w, y, z) \stackrel{\mathrm{D}}{=} \mathbb{E}(\xi(x) \mid w, y, z)$. By means of the conditional Gaussian calculations [2, Chapter 5.5], we have that $\partial \overline{\mathbb{E}}\left(x_{*} ; w, y, z\right)=y$, $\partial^{2} \overline{\mathbb{E}}\left(x_{*} ; w, y, z\right)=-\Delta(u-z), \partial^{3} \overline{\mathbb{E}}\left(x_{*} ; w, y, z\right)=-(A / \Delta) y$, and $\partial^{4} \overline{\mathbb{E}}\left(x_{*} ; w, y, z\right)=A u+$ 
$O(z)$, where ' $\partial$ ' is the partial derivative with respect to $x$. We perform a Taylor expansion on $\overline{\mathbb{E}}(x ; w, y, z)$. Using the notation $\vartheta(x)=O\left(u^{1 / 2+4 \delta} x^{4}+u x^{6}\right)$, we obtain that on the set $\mathcal{L}_{u}$

$$
\begin{aligned}
\xi(x)= & u+w+y\left(x-x_{*}\right)-\frac{\Delta(u-z)}{2}\left(x-x_{*}\right)^{2} \\
& -\frac{A}{6 \Delta} y\left(x-x_{*}\right)^{3}+\frac{A u}{24}\left(x-x_{*}\right)^{4}+g\left(x-x_{*}\right)+\vartheta\left(x-x_{*}\right) \\
= & u+w+\frac{y^{2}}{2 \Delta(u-z)}-\frac{\Delta(u-z)}{2}\left(x-x_{*}-\frac{y}{\Delta(u-z)}\right)^{2} \\
& -\frac{A}{6 \Delta} y\left(x-x_{*}\right)^{3}+\frac{A u}{24}\left(x-x_{*}\right)^{4}+g\left(x-x_{*}\right)+\vartheta\left(x-x_{*}\right) .
\end{aligned}
$$

For $\delta>0$, we further localize the event by the following proposition.

Proposition 2. For each $\delta, \delta^{\prime}>0$ chosen to be small enough and $\delta^{\prime}>24 \delta$, we have that

$$
\mathbb{P}\left(\sup _{|x|>u^{-1 / 2+8 \delta}}\left(|g(x)|-\delta^{\prime} u x^{2}\right)>0 \quad \text { or } \sup _{|x| \leq u^{-1 / 2+8 \delta}}|g(x)|>u^{-1 / 2+\delta^{\prime}}, \mathcal{L}_{u}\right)=o\left(u^{-1} \mathrm{e}^{-u^{2} / 2}\right) \text {. }
$$

With this proposition, let

$$
\mathcal{L}_{u}^{\prime}=\mathcal{L}_{u} \cap\left\{\sup _{|x|>u^{-1 / 2+8 \delta}}\left[|g(x)|-\delta^{\prime} u x^{2}\right]<0\right\} \cap\left\{\sup _{|x| \leq u^{-1 / 2+8 \delta}}|g(x)|<u^{-1 / 2+\delta^{\prime}}\right\} .
$$

We further reduce the event to $\Delta \int_{\mathscr{L}_{u}} \mathbb{P}\left(\mathbb{E}_{1}, \mathcal{L}_{u}^{\prime} \mid w, y, z\right) h(w, y, z) \mathrm{d} w \mathrm{~d} y \mathrm{~d} z$.

\section{Step 1: $v^{\prime}(x)$}

It is necessary to be reminded that the derivations are on the set $\mathcal{L}_{u}^{\prime}$. Consider the change of variable that $s=s(x): x \rightarrow \sqrt{\Delta(u-z)}\left(x-x_{*}-(y / \Delta(u-z))\right)$. We insert $s$ into the expansion in (11) and obtain

$$
\begin{aligned}
\xi(x)= & u+w+\frac{y^{2}}{2 \Delta(u-z)}-\frac{A y^{4}}{8 \Delta^{4}(u-z)^{3}}-\frac{s^{2}}{2}-\frac{A y^{3}}{3 \Delta^{7 / 2}(u-z)^{5 / 2}} s \\
& -\frac{A y^{2}}{4 \Delta^{3}(u-z)^{2}} s^{2}+\frac{A}{24 \Delta^{2}(u-z)} s^{4}+g\left(x-x_{*}\right)+\vartheta\left(x-x_{*}\right)+o\left(s^{4} u^{-5 / 4}\right) .
\end{aligned}
$$

Initially we are interested in approximating

$$
F(x)-\frac{\int_{0}^{L} F(t) \mathrm{e}^{\sigma \xi(t)} \mathrm{d} t}{\int_{0}^{L} \mathrm{e}^{\sigma \xi(t)} \mathrm{d} t}=\frac{\int_{0}^{L}(F(x)-F(t)) \mathrm{e}^{\sigma \xi(t)} \mathrm{d} t}{\int_{0}^{L} \mathrm{e}^{\sigma \xi(t)} \mathrm{d} t} .
$$

To compute the integration it is convenient to write the terms in the above expansion formula for $\xi(x)$, which do not include $x$ (or equivalently $s$ ), as

$$
c_{*} \stackrel{\mathrm{D}}{=} \sigma\left[u+w+\frac{y^{2}}{2 \Delta(u-z)}-\frac{A y^{4}}{8 \Delta^{4}(u-z)^{3}}\right] .
$$

We first consider the denominator

$$
\begin{aligned}
\int_{0}^{L} \mathrm{e}^{\sigma \xi(x)} \mathrm{d} x= & \mathrm{e}^{c_{*}} \int_{0}^{L} \exp \left\{\sigma \left[-\frac{s^{2}}{2}-\frac{A y^{3}}{3 \Delta^{7 / 2}(u-z)^{5 / 2}} s-\frac{A y^{2}}{4 \Delta^{3}(u-z)^{2}} s^{2}\right.\right. \\
& \left.\left.+\frac{A}{24 \Delta^{2}(u-z)} s^{4}+g\left(x-x_{*}\right)+\vartheta\left(x-x_{*}\right)\right]\right\} \mathrm{d} x,
\end{aligned}
$$


and separate it into two parts

$$
\int_{0}^{L} \mathrm{e}^{\sigma \xi(x)} \mathrm{d} x=\int_{\left|x-x_{*}\right|<u^{-1 / 2+8 \delta}} \mathrm{e}^{\sigma \xi(x)} \mathrm{d} x+\int_{\left|x-x_{*}\right| \geq u^{-1 / 2+8 \delta}} \mathrm{e}^{\sigma \xi(x)} \mathrm{d} x=J_{1}+J_{2} .
$$

According to Assumption 2 and on the set $\left\{\sup _{|x|>u^{-1 / 2+8 \delta}}\left[|g(x)|-\delta^{\prime} u x^{2}\right] \leq 0\right\}$ ( $\delta^{\prime}$ can be chosen arbitrarily small), there exists some $\varepsilon_{0}>0$ so that the minor term

$$
\begin{aligned}
J_{2} & =\int_{\left|x-x_{*}\right| \geq u^{-1 / 2+8 \delta}} \exp (\sigma \xi(x)) \mathrm{d} x \\
& \leq \int_{\left|x-x_{*}\right| \geq u^{-1 / 2+8 \delta}} \exp \left(c_{*}-2 \varepsilon_{0} u\left(x-x_{*}\right)^{2}\right) \\
& \leq \exp \left(c_{*}-\varepsilon_{0} u^{16 \delta}\right) .
\end{aligned}
$$

We now proceed to the dominating term $J_{1}$. Note that, on the set $\left|x-x_{*}\right|<u^{-1 / 2+8 \delta}$, $\vartheta\left(x-x_{*}\right)=o\left(u^{-1}\right)$. Then, we obtain

$$
\begin{aligned}
J_{1}= & \frac{\mathrm{e}^{c_{*}+o\left(u^{-1}\right)}}{\sqrt{\Delta(u-z)}} \mathrm{e}^{\omega(u)} \int_{\left|x-x_{*}\right|<u^{-1 / 2+8 \delta}} \exp \left\{\sigma \left[-\frac{s^{2}}{2}-\frac{A y^{3}}{3 \Delta^{7 / 2}(u-z)^{5 / 2}} s\right.\right. \\
& \left.\left.-\frac{A y^{2}}{4 \Delta^{3}(u-z)^{2}} s^{2}+\frac{A}{24 \Delta^{2}(u-z)} s^{4}\right]\right\} \mathrm{d} s,
\end{aligned}
$$

where $\omega(u)=O\left(\sup _{|x| \leq u^{-1 / 2+8 \delta}}|g(x)|\right)$. Since $\operatorname{var}(g(x))=O\left(|x|^{6}\right)$ it is helpful to keep in mind that $\omega(u)=O_{p}\left(u^{-3 / 2+24 \delta}\right)$.

Lemma 1. On the set $\mathcal{L}_{u}^{\prime}$, we have that

$$
\begin{aligned}
& \int_{\left|x-x_{*}\right|<u^{-1 / 2+8 \delta}} \exp \left(\sigma \left[-\frac{s^{2}}{2}-\frac{A y^{3}}{3 \Delta^{7 / 2}(u-z)^{5 / 2}} s\right.\right. \\
& \left.\left.-\frac{A y^{2}}{4 \Delta^{3}(u-z)^{2}} s^{2}+\frac{A}{24 \Delta^{2}(u-z)} s^{4}\right]\right) \mathrm{d} s \\
& =\sqrt{\frac{2 \pi}{\sigma}} \exp \left(-\frac{A y^{2}}{4 \Delta^{3}(u-z)^{2}}+\frac{A}{8 \Delta^{2} \sigma u}+o\left(u^{-1}\right)\right) .
\end{aligned}
$$

We substitute the result of the above lemma into the expression for the $J_{1}$ term, combine the $J_{1}$ and $J_{2}$ terms, to obtain that, on the set $\mathcal{L}_{u}^{\prime}$

$$
\begin{aligned}
\int_{0}^{L} \mathrm{e}^{\sigma \xi(x)} \mathrm{d} x= & \sqrt{\frac{2 \pi}{\sigma \Delta(u-z)}} \\
& \times \exp \left\{c_{*}-\frac{A y^{2}}{4 \Delta^{3}(u-z)^{2}}+\frac{A}{8 \Delta^{2} \sigma(u-z)}+\omega(u)+o\left(u^{-1}\right)\right\} .
\end{aligned}
$$

We now proceed to the analysis of (12). Let $\tau_{*}=x_{*}+\gamma_{*}$, where $\gamma_{*}=u^{-1 / 2} \Delta^{-1 / 2} \sigma^{-1 / 2}$. For each $x-\tau_{*}=O\left(u^{-1 / 2+16 \delta}\right)$, we define a change of variable so that $\gamma=x-x_{*}-(y / \Delta(u-z))$. Note that $\xi(x)$ is approximately a quadratic function with a maximum at $x_{*}+(y / \Delta(u-z))$. Thus, $\gamma$ is approximately the distance to the mode of $\xi(x)$. Similar to the derivations of Lemma 1 and using the results in (13) the following lemma provides an approximation of (12). 
Lemma 2. On the set $\mathcal{L}_{u}^{\prime}$, we have that

$$
\begin{aligned}
F(x)-\frac{\int_{0}^{L} F(t) \mathrm{e}^{\sigma \xi(t)} \mathrm{d} t}{\int_{0}^{L} \mathrm{e}^{\sigma \xi(t)} \mathrm{d} t}= & p(x) \gamma \exp \left\{-\frac{p^{\prime}(x)}{2 p(x) \gamma}\left(\gamma^{2}+\frac{1}{\sigma \Delta(u-z)}\right)\right. \\
& +\frac{p^{\prime \prime}(x)}{6 p(x)}\left(\gamma^{2}+\frac{3}{\sigma \Delta(u-z)}\right)+\frac{A y^{3}}{3 \Delta^{4}(u-z)^{3} \gamma} \\
& \left.+o\left(u^{-1}\right)+\omega(u)\right\} .
\end{aligned}
$$

We apply the change of variable $\gamma=x-x_{*}-(y / \Delta(u-z))$ to the representation of $\xi(x)$ in (11) and obtain that

$$
\begin{aligned}
\xi(x)= & u+w+\frac{y^{2}}{2 \Delta(u-z)}-\frac{\Delta(u-z)}{2} \gamma^{2}-\frac{A}{6 \Delta} y\left(\gamma+\frac{y}{\Delta(u-z)}\right)^{3} \\
& +\frac{A u}{24}\left(\gamma+\frac{y}{\Delta(u-z)}\right)^{4}+g\left(x-x_{*}\right)+\vartheta\left(x-x_{*}\right) .
\end{aligned}
$$

We now combine (14) and (15) and obtain that for $\left|x-x_{*}\right| \leq u^{-1 / 2+8 \delta}$

$$
\begin{aligned}
v^{\prime}(x)= & \exp \left(\sigma u+\sigma w+\frac{\sigma y^{2}}{2 \Delta(u-z)}\right) \times p(x) \gamma \exp \left(-\frac{\sigma \Delta u}{2} \gamma^{2}\right) \\
& \times \exp \left\{\frac{\sigma \Delta z}{2} \gamma^{2}-\frac{\sigma A}{6 \Delta} y\left(\gamma+\frac{y}{\Delta(u-z)}\right)^{3}+\frac{\sigma A u}{24}\left(\gamma+\frac{y}{\Delta(u-z)}\right)^{4}\right. \\
& -\frac{p^{\prime}(x)}{2 p(x) \gamma}\left(\gamma^{2}+\frac{1}{\sigma \Delta(u-z)}\right)+\frac{p^{\prime \prime}(x)}{6 p(x)}\left(\gamma^{2}+\frac{3}{\sigma \Delta(u-z)}\right) \\
& \left.+\frac{A y^{3}}{3 \Delta^{4}(u-z)^{3} \gamma}+o\left(u^{-1}\right)+\omega(u)\right\}
\end{aligned}
$$

Step 2: the event $\mathbb{E}_{1}=\left\{\max _{x \in\left[u^{-1 / 2+\delta}, L-u^{-1 / 2+\delta}\right]}\left|v^{\prime}(x)\right|>b\right\}$

By the definition of $u$ and the analytic form of (16), we have that

$$
v^{\prime}(x) \geq b=p\left(x_{*}\right) \gamma_{*} \mathrm{e}^{\sigma u-(\Delta \sigma u / 2) \gamma_{*}^{2}}
$$

if and only if $\gamma>0$ and

$$
\begin{aligned}
\sigma w & +\frac{\sigma y^{2}}{2 \Delta(u-z)}+\frac{\sigma \Delta z}{2} \gamma^{2}-\frac{\sigma A}{6 \Delta} y\left(\gamma+\frac{y}{\Delta(u-z)}\right)^{3}+\frac{\sigma A u}{24}\left(\gamma+\frac{y}{\Delta(u-z)}\right)^{4} \\
& -\frac{p^{\prime}(x)}{2 p(x) \gamma}\left(\gamma^{2}+\frac{1}{\sigma \Delta(u-z)}\right)+\frac{p^{\prime \prime}(x)}{6 p(x)}\left(\gamma^{2}+\frac{3}{\sigma \Delta(u-z)}\right) \\
& +\frac{A y^{3}}{3 \Delta^{4}(u-z)^{3} \gamma}+\log H(\gamma, u)-\log H\left(\gamma_{*}, u\right)+\log \frac{p(x)}{p\left(x_{*}\right)} \geq o\left(u^{-1}\right)-\omega(u),
\end{aligned}
$$

where $H$ is defined as in (5) and $\gamma_{*}=(1 / \sqrt{\sigma \Delta u})$. We write the left-hand side of the above display as $R(\gamma)+\log H(\gamma, u)-\log H\left(\gamma_{*}, u\right)$. Note that $\partial_{\gamma}^{2} \log H\left(\gamma_{*}, u\right)=-2 \Delta \sigma u$ and the derivative of the remainder term is $\partial_{\gamma} R\left(\gamma_{*}\right)=o(1)+O\left(z \gamma_{*}\right)$. Thus, $\log H(\gamma, u)$ dominates the variation. In particular, the left-hand side of (17) is maximized at $\gamma=\gamma_{*}+o\left(u^{-1}\right)+O\left(z \gamma_{*} / u\right)=$ $\left(1 / \sqrt{u \Delta \sigma}+o\left(u^{-1}\right)+O\left(z \gamma_{*} / u\right)\right.$, equivalently, at $x=x_{*}+\gamma_{*}+y / \Delta(u-z)+o\left(u^{-1}\right)+$ 
$O\left(z \gamma_{*} / u\right)$. Therefore, $\max _{|\gamma| \leq u^{-1 / 2+8 \delta}} R(\gamma)+\log H(\gamma, u)-\log H\left(\gamma_{*}, u\right)=R\left(\gamma_{*}\right)+o\left(u^{-1}\right)+$ $O\left(z^{2} / u^{2}\right)$. This is interpreted as $\max _{\left|x-x_{*}\right| \leq u^{-1 / 2+8 \delta}} v^{\prime}(x) \geq b$ if and only if

$$
\begin{aligned}
\mathcal{A} \stackrel{\mathrm{D}}{=} & \sigma w+\frac{\sigma y^{2}}{2 \Delta(u-z)}+\frac{\sigma \Delta z}{2} \gamma_{*}^{2}-\frac{\sigma A}{6 \Delta} y\left(\gamma_{*}+\frac{y}{\Delta(u-z)}\right)^{3} \\
& +\frac{\sigma A u}{24}\left(\gamma_{*}+\frac{y}{\Delta(u-z)}\right)^{4}-\frac{p^{\prime}(x)}{2 p(x) \gamma_{*}}\left(\gamma_{*}^{2}+\frac{1}{\sigma \Delta(u-z)}\right) \\
& +\frac{p^{\prime \prime}(x)}{6 p(x)}\left(\gamma_{*}^{2}+\frac{3}{\sigma \Delta(u-z)}\right)+\frac{A y^{3}}{3 \Delta^{4}(u-z)^{3} \gamma_{*}} \\
& +\log \frac{p\left(x_{*}+\gamma_{*}+\Delta^{-1}(u-z)^{-1} y\right)}{p\left(x_{*}\right)}+O\left(z^{2} / u^{2}\right) \\
\geq & o\left(u^{-1}\right)-\omega(u) .
\end{aligned}
$$

Note that on the region $\left|x-x_{*}\right|>u^{-1 / 2+8 \delta}$ we need to consider the variation of $g\left(x-x_{*}\right)$. On the set $\mathcal{L}_{u}^{\prime}$, the variation of $v^{\prime}(x)$ is dominated by $\log H(\gamma, u)$. In particular, on the set $\left|x-x_{*}\right|>u^{-1 / 2+8 \delta}$ we have $\log H(\gamma, u)-\log H\left(\gamma_{*}, u\right) \leq-\varepsilon_{0} u\left(\gamma-\gamma_{*}\right)^{2}$. Furthermore, on the set $\mathcal{L}_{u}^{\prime}$, we have that $\sup _{|x|>u^{-1 / 2+8 \delta}}\left(|g(x)|-\delta^{\prime} u x^{2}\right)<0$. We can choose $\delta^{\prime}<\varepsilon_{0} / 2$, then $2|g(x)|<\log H\left(\gamma_{*}, u\right)-\log H(\gamma, u)$ for all $\left|x-x_{*}\right|>u^{-1 / 2+8 \delta}$. Thus, on the set $\mathcal{L}_{u}^{\prime}$, the maximum of $v^{\prime}(x)$ is attained on $\left|x-x_{*}\right| \leq u^{-1 / 2+8 \delta}$, i.e. $\max _{\left[u^{-1 / 2+\delta}, L-u^{-1 / 2+\delta}\right]} v^{\prime}(x)>b$ if and only if $\mathcal{A}>o\left(u^{-1}\right)-\omega(u)$. The following lemma simplifies the analytic form of $\mathcal{A}$.

Lemma 3. The expression $\mathcal{A}$ can be simplified to

$$
\begin{aligned}
\mathcal{A}= & \sigma w+\frac{\sigma y^{2}}{2 \Delta u}+\frac{\sigma}{2 \Delta u^{2}} y^{2} z+\frac{z}{2 u}+\frac{A}{24 \sigma \Delta^{2} u} \\
& +\frac{p^{\prime \prime}\left(x_{*}\right)}{6 p\left(x_{*}\right) \sigma \Delta u}-\frac{\sigma A y^{4}}{8 \Delta u^{3}}+\frac{y^{2}}{u^{2}}\left(-\frac{A}{4 \Delta^{3}}+\frac{p^{\prime \prime}\left(x_{*}\right)}{2 p\left(x_{*}\right) \Delta^{2}}\right) \\
& +o\left(u^{-1}+y^{2} u^{-2}\right)+O\left(z^{2} / u^{2}\right) .
\end{aligned}
$$

With exactly the same development we have $\max _{x \in\left[u^{-1 / 2+\delta}, L-u^{-1 / 2+\delta}\right]}\left[-v^{\prime}(x)\right] \geq b$ if and only if $\mathcal{A} \geq o\left(u^{-1}\right)+\omega(u)$. In fact, from the technical proof of Lemma 3, we basically choose $\gamma=-\gamma_{*}+o\left(u^{-1}\right)+O\left(z \gamma_{*} / u\right)$ and all the other derivations are the same. We omit the repetitive details. Thus, the event $\mathbb{E}_{1}$ occurs if and only if $\mathcal{A} \geq o\left(u^{-1}\right)+\omega(u)$.

\section{Step 3: evaluation of the integral in (10)}

Lemma 4. The density of $\left(\xi(x), \xi^{\prime \prime}(x), \xi^{\prime \prime}(x)\right)$ evaluated at $(u+w, y,-\Delta(u-z))$ is

$$
h(w, y, z)=\frac{\mathrm{e}^{-S(w, y, z) / 2}}{(2 \pi)^{3 / 2} \sqrt{\Delta\left(A-\Delta^{2}\right)}},
$$

where

$$
S(w, y, z)=u^{2}+w^{2}+\frac{\Delta^{2}(w+z)^{2}}{A-\Delta^{2}}+2 u\left(w+\frac{y^{2}}{2 \Delta u}\right) .
$$

The proof of the above lemma is elementary and therefore is omitted; see also Chapter 5.5 in [2]. 
We insert the expression of $\mathcal{A}$ in Lemma 3 to the exponent of the density function

$$
\begin{aligned}
S(w, y, z)= & u^{2}+w^{2}+\frac{\Delta^{2}(w+z)^{2}}{A-\Delta^{2}}+2 u\left[\frac{\mathcal{A}}{\sigma}-\frac{y^{2} z}{2 \Delta u^{2}}-\frac{z}{2 \sigma u}-\frac{A}{24 \sigma^{2} \Delta^{2} u}\right. \\
& -\frac{p^{\prime \prime}\left(x_{*}\right)}{6 p\left(x_{*}\right) \sigma^{2} \Delta u}+\frac{A y^{4}}{8 \Delta^{4} u^{3}}-\frac{y^{2}}{u^{2}}\left(-\frac{A}{4 \sigma \Delta^{3}}+\frac{p^{\prime \prime}\left(x_{*}\right)}{2 p\left(x_{*}\right) \sigma \Delta^{2}}\right) \\
& \left.+o\left(u^{-1}+y^{2} u^{-2}\right)+O\left(z^{2} / u^{2}\right)\right] .
\end{aligned}
$$

The following lemma provides a lower bound of $S(w, y, z)$ for the dominated convergence theorem.

Lemma 5. On the set $\mathcal{L}_{u}^{\prime}$

$$
\begin{aligned}
S(w, y, z) \geq & u^{2}+2 u \mathcal{A} / \sigma+\frac{\Delta^{2}}{A}\left(\frac{A}{2 \Delta^{3}} \frac{y^{2}}{u}-z\right)^{2} \\
& +\frac{1+o(1)}{\sigma}\left(\frac{A}{2 \Delta^{3}} \frac{y^{2}}{u}-z\right)-\frac{p^{\prime \prime}\left(x_{*}\right)}{p\left(x_{*}\right) \sigma \Delta^{2}} \frac{y^{2}}{u}+O(1) .
\end{aligned}
$$

It is useful to keep in mind that $p^{\prime \prime}\left(x_{*}\right)<0$. Let $\mathcal{A}_{u}=u \mathcal{A}$. Note that for each fixed $\left(\mathcal{A}_{u}, y, z\right), w \rightarrow 0$ as $u \rightarrow \infty$. Furthermore, note that $\omega(u)=O\left(\sup _{|x| \leq u^{-1 / 2+8 \delta}}|g(x)|\right)=$ $O_{p}\left(u^{-3 / 2+24 \delta}\right)$. We consider a change of variable from $(w, y, z)$ to $\left(\mathcal{A}_{u}, y, z\right)$. The dominated convergence theorem and (18) yield that

$$
\begin{array}{rl}
\Delta \int_{\mathscr{L}_{u}} & \mathbb{P}\left(\mathbb{E}_{1}, \mathcal{L}_{u}^{\prime} \mid w, y, z\right) h(w, y, z) \mathrm{d} w \mathrm{~d} y \mathrm{~d} z \\
= & \frac{\sqrt{\Delta}}{(2 \pi)^{3 / 2} \sqrt{A-\Delta^{2}}} \times \int_{\mathcal{L}_{u}} \mathbb{P}\left(\mathcal{A}>\omega(u), \mathcal{L}_{u}^{\prime} \mid w, y, z\right) \mathrm{e}^{-(1 / 2) S(w, y, z)} \mathrm{d} w \mathrm{~d} y \mathrm{~d} z \\
& \sim \frac{\sqrt{\Delta}}{(2 \pi)^{3 / 2} \sqrt{A-\Delta^{2}}} \times \int_{\mathscr{L}_{u}} I\left(\mathcal{A}_{u}>0\right) \mathrm{e}^{-(1 / 2) S(w, y, z)} \frac{\mathrm{d} \mathcal{A}_{u}}{\sigma u} \mathrm{~d} y \mathrm{~d} z .
\end{array}
$$

For the last step we use the fact that $\mathbb{P}\left(\mathcal{L}_{u}^{\prime} \mid w, y, z\right) \rightarrow 1$ and $\mathbb{P}\left(\mathcal{A}>\omega(u), \mathcal{L}_{u}^{\prime} \mid w, y, z\right) \rightarrow$ $I\left(\mathcal{A}_{u}>0\right)$ as $u \rightarrow \infty$. We insert the expression $S(w, y, z)$ from (18) and set $w=0$ (by the dominated convergence theorem and the fact that for fixed $\mathcal{A}_{u}, y$, and $z$, we have $w \rightarrow 0$ as $u \rightarrow \infty)$, giving

$$
\begin{aligned}
\sim & \frac{\sqrt{\Delta}}{(2 \pi)^{3 / 2} \sqrt{A-\Delta^{2}}} u^{-1} \exp \left(-u^{2} / 2+\frac{A}{24 \sigma^{2} \Delta^{2}}+\frac{p^{\prime \prime}\left(x_{*}\right)}{6 p\left(x_{*}\right) \sigma^{2} \Delta}\right) \int_{0}^{\infty} \frac{1}{\sigma} \mathrm{e}^{-\mathcal{A}_{u} / \sigma} \mathrm{d} \mathcal{A}_{u} \\
& \times \int \exp \left(-\frac{1}{2}\left[\frac{\Delta^{2} z^{2}}{A-\Delta^{2}}-\frac{z}{\sigma}-\frac{y^{2} z}{\Delta u}+\frac{A}{4 \Delta^{4}} \frac{y^{4}}{u^{2}}\right.\right. \\
& \left.\left.-\frac{y^{2}}{u}\left(-\frac{A}{2 \sigma \Delta^{3}}+\frac{p^{\prime \prime}\left(x_{*}\right)}{p\left(x_{*}\right) \sigma \Delta^{2}}\right)\right]\right) \mathrm{d} y \mathrm{~d} z .
\end{aligned}
$$

We apply the change of variable that $y_{u}=(y / \sqrt{u})$ for the integration, giving

$$
\sim D u^{-1 / 2} \mathrm{e}^{-u^{2} / 2}
$$

This corresponds to the first term of the approximation in the statement of the theorem. 


\subsection{The approximation of $\mathbb{P}\left(\mathbb{E}_{3}\right)$}

The analysis of $\mathbb{P}\left(\mathbb{E}_{2}\right)$ and $\mathbb{P}\left(\mathbb{E}_{3}\right)$ are analogous. We only need to derive $\mathbb{P}\left(\mathbb{E}_{3}\right)$. The difference between the analyses of $\mathbb{P}\left(\mathbb{E}_{3}\right)$ and $\mathbb{P}\left(\mathbb{E}_{1}\right)$ is that the integrals in the factor (12) are truncated by the boundary and therefore most of the calculations are related to conditional Gaussian distributions. We redefine some notation. Let $u_{L}$ and $\zeta_{L}$ be defined as in Section 2 prior to the statement of the theorem. We first define $t_{L}=L-\left(\zeta_{L} / \sqrt{\Delta \sigma u_{L}}\right)$ as the location where $\xi(x)$ is likely to have a high excursion given that $v^{\prime}(x)$ has a high excursion at the right boundary $L$. We will perform a Taylor expansion by conditioning on the field at $t_{L}$. We redefine the notation $(w, y, z)$ as $\xi\left(t_{L}\right)=u_{L}+w, \xi^{\prime}\left(t_{L}\right)=y$, and $\xi^{\prime \prime}\left(t_{L}\right)=-\Delta\left(u_{L}-z\right)$. Furthermore, we consider the following change of variables ' $\gamma$ ' and ' $s$ '

$$
x=\gamma+t_{L}+\frac{y}{\Delta\left(u_{L}-z\right)}, \quad t=t_{L}+\frac{y}{\Delta\left(u_{L}-z\right)}+\frac{s}{\sqrt{\Delta\left(u_{L}-z\right)}} .
$$

With some simple calculations we arrive at $t \leq L$ if and only if

$$
s \leq \sqrt{\frac{\left(1-z / u_{L}\right)}{\sigma}} \zeta_{L}-\frac{y}{\sqrt{\Delta\left(u_{L}-z\right)}} .
$$

Furthermore, it is useful to keep in mind that $v^{\prime}(x)$ is maximized when $\gamma$ is of order $\left(1 / \sqrt{u_{L}}\right)$. Let $g(x)$ be the remainder process such that $\xi(x)=\mathbb{E}(\xi(x) \mid w, y, z)+g\left(x-t_{L}\right)$. Similar to the analysis of $\mathbb{P}\left(\mathbb{E}_{1}\right)$, we first localize the event via the following proposition.

Proposition 3. Using the notation in Theorem 1, under Assumptions 1 and 2, consider

$$
\begin{aligned}
\mathcal{C}_{u_{L}}=\{|w|> & \left.u_{L}^{3 \delta}\right\} \cup\left\{|y|>u_{L}^{1 / 2+4 \delta}\right\} \cup\left\{|z|>u_{L}^{1 / 2+4 \delta}\right\} \\
& \cup\left\{\sup _{|x|>u_{L}^{-1 / 2+8 \delta}}\left[|g(x)|-\delta^{\prime} u_{L} x^{2}\right]>0\right\} \cup\left\{\sup _{|x| \leq u_{L}^{-1 / 2+8 \delta}}|g(x)|>u_{L}^{-1 / 2+\delta^{\prime}}\right\} .
\end{aligned}
$$

Then, for any $\delta>0$ and $\delta^{\prime}>24 \delta$, we have that $\mathbb{P}\left(\mathcal{C}_{u_{L}} ; \mathbb{E}_{3}\right)=o\left(u_{L}^{-1} \mathrm{e}^{-u_{L}^{2} / 2}\right)$.

Let $\mathcal{L}_{u_{L}}^{*}=\mathcal{C}_{u_{L}}^{c}$ and we only need to consider $\mathbb{P}\left(\mathcal{L}_{u_{L}}^{*}, \mathbb{E}_{3}\right)$. With a similar derivation as that for $\mathbb{P}\left(\mathbb{E}_{1}\right)$, the following lemma provides an estimate of

$$
\int_{0}^{L}(F(x)-F(t)) \mathrm{e}^{(\sigma \xi(t))} \mathrm{d} t / \int_{0}^{L} \mathrm{e}^{(\sigma \xi(t))} \mathrm{d} t
$$

Lemma 6. On the set $\mathcal{L}_{u_{L}}^{*}$, we have that

$$
\begin{aligned}
\frac{\int_{0}^{L}(F(x)-F(t)) \mathrm{e}^{\sigma \xi(t)} \mathrm{d} t}{\int_{0}^{L} \mathrm{e}^{\sigma \xi(t)} \mathrm{d} t} \\
=\frac{1}{\sqrt{\Delta \sigma u_{L}}} \exp \left(\frac{z}{2 u_{L}}-\frac{A}{24 \Delta^{2} \sigma u_{L}} \mathbb{E}\left[Z^{4} \mid Z \leq \zeta_{L}\right]+\lambda\left(u_{L}\right)+\omega\left(u_{L}\right)\right) \\
\quad \times\left\{\mathbb { E } \left[p(x)\left(\gamma \sqrt{\sigma \Delta\left(u_{L}-z\right)}-Z\right)-\frac{p^{\prime}(x)}{2 \sqrt{\sigma \Delta u_{L}}}\left(\gamma \sqrt{\sigma \Delta\left(u_{L}-z\right)}\right.\right.\right. \\
\left.\quad-Z)^{2} \mid Z \leq \sqrt{1-\frac{z}{u_{L}}} \zeta_{L}-\sqrt{\frac{\sigma}{\Delta\left(u_{L}-z\right)}} y\right]
\end{aligned}
$$




$$
\begin{aligned}
& +\mathbb{E}\left[\frac{p^{\prime \prime}(x)}{6 \sigma \Delta u_{L}}\left(\gamma \sqrt{\sigma \Delta u_{L}}-Z\right)^{3}+\frac{A p(x)}{24 \Delta^{2} \sigma^{2} u_{L}} Z^{4}\left(\gamma \sqrt{\sigma \Delta u_{L}}\right.\right. \\
& \left.\left.-Z) \mid Z \leq \sqrt{1-\frac{z}{u_{L}}} \zeta_{L}-\sqrt{\frac{\sigma}{\Delta\left(u_{L}-z\right)}} y\right]\right\}
\end{aligned}
$$

where

$$
\begin{aligned}
\lambda\left(u_{L}\right) & =O\left(y^{3} / u_{L}^{5 / 2}+y^{2} / u_{L}^{2}+y / u^{3 / 2}\right)+o\left(u_{L}^{-1}+u_{L}^{-1} z\right), \\
\omega(u) & =O\left(\sup _{|x| \leq u^{-1 / 2+8 \delta}}|g(x)|\right),
\end{aligned}
$$

and $Z$ is a standard Gaussian random variable.

Inside the ' \{\} ' of the above approximation, the first expectation term is the dominating term and the second term is of order $o\left(u^{-1}\right)$. The next lemma presents an approximation of $v^{\prime}(x)$.

Lemma 7. On the set $\mathcal{L}_{u_{L}}^{*}$, we have that

$$
\begin{aligned}
v^{\prime}(x)= & \exp \left(\lambda\left(u_{L}\right)+o\left(y u_{L}^{-1}\right)+O\left(y^{2} z u_{L}^{-2}\right)+\omega\left(u_{L}\right)+\sigma u_{L}+\sigma w+\frac{\sigma y^{2}}{2 \Delta u_{L}}+\frac{A \sigma u_{L}}{24} \gamma^{4}\right) \\
& \times \frac{1}{\sqrt{\Delta \sigma u_{L}}} \exp \left(\frac{z}{2 u_{L}}-\frac{A}{24 \Delta^{2} \sigma u_{L}} \mathbb{E}\left[Z^{4} \mid Z \leq \zeta_{L}\right]\right) \\
& \times H_{L, x}\left(\gamma \sqrt{\sigma \Delta\left(u_{L}-z\right)}, \sqrt{1-\frac{z}{u_{L}}} \zeta_{L}-\sqrt{\frac{\sigma}{\Delta\left(u_{L}-z\right)}} y ; u_{L}\right) \\
& \times \exp \left\{\frac{\mathbb{E}\left[\left(p^{\prime \prime}(x) /\left(6 \sigma \Delta u_{L}\right)\right)\left(\gamma \sqrt{\sigma \Delta u_{L}}-Z\right)^{3}+\left(A p(x) /\left(24 \Delta^{2} \sigma^{2} u_{L}\right)\right) Z^{4}\left(\gamma \sqrt{\sigma u_{L}}-Z\right) \mid Z \leq \zeta_{L}\right]}{p(x) \mathbb{E}\left[\gamma \sqrt{\sigma \Delta u_{L}}-Z \mid Z \leq \zeta_{L}\right]}\right\},
\end{aligned}
$$

where

$$
H_{L, y}(x, \zeta ; u) \stackrel{\mathrm{D}}{=} \mathrm{e}^{-\left(x^{2} / 2\right)} \times \mathbb{E}\left[p(y)(x-Z)-\frac{p^{\prime}(y)}{2 \sqrt{\Delta \sigma u}}(x-Z)^{2} \mid Z \leq \zeta\right] .
$$

Note that the definition of $H_{L, y}(x, \zeta ; z, u)$ is slightly different from $H_{L}(x, \zeta, u)$ defined as in Section 2. In particular, if we let $y=L$, then $H_{L, y}(x, \zeta ; u)=H_{L}(x, \zeta ; u)$. Furthermore, according to the change of variable in (20), $x \leq L$ if and only if

$$
\gamma \sqrt{\sigma \Delta\left(u_{L}-z\right)} \leq \sqrt{1-\frac{z}{u_{L}}} \zeta_{L}-\sqrt{\frac{\sigma}{\Delta\left(u_{L}-z\right)}} y .
$$

Thus, the maximization of $v^{\prime}(x)$ (in choosing the variable $\gamma$ ) is subject to the above constraint. According the definition of $u_{L}$ in (7) and the notation $G_{L}\left(\zeta ; u_{L}\right)=\sup _{x \leq \zeta} \log \left|H_{L}\left(x, \zeta, u_{L}\right)\right|$, we have that $\max _{x \in\left[L-u^{-1 / 2+\delta}, L\right]}\left|v^{\prime}(x)\right|>b$ if and only if

$$
\begin{aligned}
\max _{x \in\left[L-u_{L}^{-1 / 2+\delta}, L\right]} \lambda\left(u_{L}\right)+\omega\left(u_{L}\right)+o\left(y u_{L}^{-1}\right)+O\left(y^{2} z u_{L}^{-2}\right) \\
+\sigma w+\frac{\sigma y^{2}}{2 \Delta u_{L}}+\frac{A \sigma u_{L}}{24} \gamma^{4}+\frac{z}{2 u_{L}}-\frac{A \mathbb{E}\left[Z^{4} \mid Z \leq \zeta_{L}\right]}{24 \Delta^{2} \sigma u_{L}} \\
+\log \left|H_{L, x}\left(\gamma \sqrt{\sigma \Delta\left(u_{L}-z\right)}, \sqrt{1-\frac{z}{u_{L}}} \zeta_{L}-\sqrt{\frac{\sigma}{\Delta\left(u_{L}-z\right)}} y ; u_{L}\right)\right|-G_{L}\left(\zeta_{L} ; u_{L}\right) \\
+\frac{\mathbb{E}\left[\left(p^{\prime \prime} /\left(6 \sigma \Delta u_{L}\right)\right)\left(\gamma \sqrt{\sigma \Delta u_{L}}-Z\right)^{3}+\left(A p /\left(24 \Delta^{2} \sigma^{2} u_{L}\right)\right) Z^{4}\left(\gamma \sqrt{\sigma u_{L}}-Z\right) \mid Z \leq \zeta_{L}\right]}{p(x) \mathbb{E}\left[\gamma \sqrt{\sigma \Delta u_{L}}-Z \mid Z \leq \zeta_{L}\right]}>0 .
\end{aligned}
$$

We now proceed to the evaluation of $\mathbb{P}\left(\mathbb{E}_{3}\right)$ that consists of two cases. 
We first consider the case that $\left|\sqrt{1-\left(z / u_{L}\right)} \zeta_{L}-\sqrt{\sigma /\left(\Delta\left(u_{L}-z\right)\right)} y-\zeta_{L}\right| \leq \varepsilon$. Note that the major variation of the left-hand-side of (23) is dominated by

$$
\log \left|H_{L, x}\left(\gamma \sqrt{\sigma \Delta\left(u_{L}-z\right)}, \sqrt{1-\frac{z}{u_{L}}} \zeta_{L}-\sqrt{\frac{\sigma}{\Delta\left(u_{L}-z\right)}} y ; u_{L}\right)\right| .
$$

Thanks to the discussion in Remark 1 , the above expression is maximized at (subject to the constraint (22)) $\gamma \sqrt{\sigma \Delta\left(u_{L}-z\right)}=\sqrt{1-\left(z / u_{L}\right)} \zeta_{L}-\sqrt{\left(\sigma /\left(\Delta\left(u_{L}-z\right)\right)\right)} y$, that is,

$$
\gamma=\frac{\zeta_{L}}{\sqrt{\Delta \sigma u_{L}}}-\frac{y}{\Delta\left(u_{L}-z\right)} .
$$

Recall the change of variable in (20), this corresponds to $x=L$. That is, the maximum is attained on the boundary $x=L$. Then, we can replace $H_{L, x}$ in (23) by $H_{L, L}=H_{L}$. Let $\gamma_{L}=\zeta_{L} / \sqrt{\sigma \Delta u_{L}}$. For the particular choice of $\gamma$ in (24), we have that $\gamma^{4}=\gamma_{L}^{4}+o\left(y^{2} / u_{L}^{2}\right)$. We have that $\max _{x \in\left[L-u_{L}^{-1 / 2+\delta}, L\right]}\left|v_{L}^{\prime}(x)\right|>b$ if and only if $\mathcal{A} \geq \omega\left(u_{L}\right)$ where

$$
\begin{aligned}
\mathcal{A} \stackrel{\mathrm{D}}{=} & \lambda\left(u_{L}\right)+o\left(y u_{L}^{-1}\right)+O\left(y^{2} z u_{L}^{-2}\right)+\sigma w+\frac{\sigma y^{2}}{2 \Delta u_{L}} \\
& +\frac{A \sigma u_{L}}{24} \gamma_{L}^{4}+\frac{z}{2 u_{L}}-\frac{A \mathbb{E}\left[Z^{4} \mid Z \leq \zeta_{L}\right]}{24 \Delta^{2} \sigma u_{L}} \\
& +G_{L}\left(\sqrt{1-\frac{z}{u_{L}}} \zeta_{L}-\sqrt{\frac{\sigma}{\Delta\left(u_{L}-z\right)}} y ; u_{L}\right)-G_{L}\left(\zeta_{L} ; u_{L}\right) \\
& +\frac{\mathbb{E}\left[\left(p^{\prime \prime}(L) /\left(6 \sigma \Delta u_{L}\right)\right)\left(\gamma_{L} \sqrt{\sigma \Delta u_{L}}-Z\right)^{3}+\left(A p(L) /\left(24 \Delta^{2} \sigma^{2} u_{L}\right)\right) Z^{4}\left(\gamma_{L} \sqrt{\sigma \Delta u_{L}}-Z\right) \mid Z \leq \zeta_{L}\right]}{p(L) \mathbb{E}\left[\zeta_{L}-Z \mid Z \leq \zeta_{L}\right]} .
\end{aligned}
$$

Lemma 8. The expression $\mathcal{A}$ can be simplified to

$$
\begin{aligned}
\mathcal{A}= & \lambda\left(u_{L}\right)+o\left(y u_{L}^{-1}\right)+O\left(y^{2} z u_{L}^{-2}\right)+\sigma w+\frac{\sigma y^{2}}{2 \Delta u_{L}} \\
& +\frac{z}{2 u_{L}}+\frac{\kappa_{L}}{u_{L}}-\frac{\Xi_{L}+o(1)}{2}\left(\frac{\zeta_{L} z}{2 u_{L}}+\sqrt{\frac{\sigma}{\Delta\left(u_{L}-z\right)} y}\right)^{2},
\end{aligned}
$$

where $\kappa_{L}$ is given as in (8).

With the above lemma, we rewrite $S(w, y, z)$ as

$$
\begin{aligned}
S(w, y, z)= & u_{L}^{2}+w^{2}+\frac{\Delta^{2}(w+z)^{2}}{A-\Delta^{2}}+o(1)+o\left(y^{2}\right) \\
& +2 u_{L}\left[\mathcal{A} / \sigma-\frac{z}{2 \sigma u_{L}}-\frac{\kappa_{L}}{\sigma u_{L}}+\frac{\Xi_{L}+o(1)}{2 \sigma}\left(\frac{\zeta_{L} z}{2 u_{L}}+\sqrt{\frac{\sigma}{\Delta\left(u_{L}-z\right)}} y\right)^{2}\right. \\
& \left.+\lambda\left(u_{L}\right)+o\left(y u_{L}^{-1}\right)+O\left(y^{2} z u_{L}^{-2}\right)\right] .
\end{aligned}
$$

Similar to the derivation of (19), by the dominated convergence theorem, we have that

$$
\begin{aligned}
& \mathbb{P}\left(\max _{x \in\left[L-u_{L}^{-1 / 2+\delta}, L\right]}\left|v^{\prime}(x)\right|>b ; \mathcal{L}_{u_{L}}^{*} ;\left|\sqrt{1-\frac{z}{u_{L}}} \zeta_{L}-\sqrt{\frac{\sigma}{\Delta\left(u_{L}-z\right)}} y-\zeta_{L}\right| \leq \varepsilon\right) \\
& \sim \frac{\sqrt{\Delta}}{(2 \pi)^{3 / 2} \sqrt{A-\Delta^{2}}} u_{L}^{-1} \mathrm{e}^{-u_{L}^{2} / 2+\left(\kappa_{L} / \sigma\right)} \int \exp \left(-\frac{1}{2}\left(\frac{\Delta^{2} z^{2}}{A-\Delta^{2}}-\frac{z}{\sigma}+\frac{\Xi_{L}}{\Delta} y^{2}\right)\right) \mathrm{d} y \mathrm{~d} z \\
& =D_{L} u_{L}^{-1} \mathrm{e}^{-u_{L}^{2} / 2} .
\end{aligned}
$$


The following lemma presents the case that $\left|\sqrt{1-\left(z / u_{L}\right)} \zeta_{L}-\sqrt{\sigma /\left(\Delta\left(u_{L}-z\right)\right)} y-\zeta_{L}\right| \geq \varepsilon$.

Lemma 9. Under the conditions in Theorem 1, we have that

$$
\begin{aligned}
& \mathbb{P}\left(\max _{x \in\left[L-u_{L}^{-1 / 2+\delta}, L\right]}\left|v^{\prime}(x)\right|>b ; \mathcal{L}_{u_{L}}^{*}\left|\sqrt{1-\frac{z}{u_{L}}} \zeta_{L}-\sqrt{\frac{\sigma}{\Delta\left(u_{L}-z\right)}} y-\zeta_{L}\right| \geq \varepsilon\right) \\
& \quad=o(1) u_{L}^{-1} \mathrm{e}^{-u_{L}^{2} / 2} .
\end{aligned}
$$

Combining (25), Lemma 9, and the localization result in Proposition 3, we have that

$$
\mathbb{P}\left(\max _{x \in\left[L-u_{L}^{-1 / 2+\delta}, L\right]}\left|v^{\prime}(x)\right|>b\right) \sim D_{L} u_{L}^{-1} \mathrm{e}^{-u_{L}^{2} / 2} .
$$

\section{Approximation of $\mathbb{P}\left(\mathbb{E}_{2}\right)$}

The analysis of $\mathbb{P}\left(\mathbb{E}_{2}\right)$ is completely analogous. In particular, we let $t_{0}=\zeta_{0} / \sqrt{\Delta \sigma u_{0}}$, $\xi\left(t_{0}\right)=u_{0}+w, \xi^{\prime}\left(t_{0}\right)=y$, and $\xi^{\prime \prime}\left(t_{0}\right)=-\Delta(u-z)$ and further adopt the change of variables $x=t_{0}+\left(y / \Delta\left(u_{0}-z\right)\right)-\gamma$ and $t=t_{0}+\left(y / \Delta\left(u_{0}-z\right)\right)-\left(s / \sqrt{\Delta\left(u_{0}-z\right)}\right)$. Then the calculations are exactly the same as those of $\mathbb{P}\left(\mathbb{E}_{3}\right)$. Therefore, we omit the repetitive derivations and provide the result that $\mathbb{P}\left(\max _{x \in\left[0, u_{L}^{-1 / 2+\delta}\right]}\left|v^{\prime}(x)\right|>b\right) \sim\left(D_{0} / u_{0}\right) \exp \left(-u_{0}^{2} / 2\right)$. With the inclusion-exclusion formula and $(\stackrel{x}{9})$, we conclude the proof.

\section{Acknowledgement}

This research is supported in part by NSF CMMI-1069064, NSF SES-1323977, and Army Grant W911NF-14-1-0020.

\section{References}

[1] AdLer, R. J. (1981). The Geometry of Random Fields. John Wiley, Chichester.

[2] Adler, R. J. And Taylor, J. E. (2007). Random Fields and Geometry. Springer, New York.

[3] Adler, R. J., Blanchet, J. And LiU, J. (2008). Efficient simulation for tail probabilities of Gaussian random fields. In Proc. Winter Simulation Conf. 2008, IEEE, New York, pp. 328-336.

[4] Adler, R. J., Blanchet, J. and Liu, J. (2012). Efficient Monte Carlo for high excursions of Gaussian random fields. Ann. Appl. Prob. 22, 1167-1214.

[5] AzAïs, J.-M. AND WsCHEBOR, M. (2008). A general expression for the distribution of the maximum of a Gaussian field and the approximation of the tail. Stoch. Process. Appl. 118, 1190-1218. (Erratum: 120(2010), 2100-2101.)

[6] Borell, C. (2003). The Ehrhard inequality. C. R. Math. Acad. Sci. Paris 337, 663-666.

[7] LiU, J. (2012). Tail approximations of integrals of Gaussian random fields. Ann. Prob. 40, 1069-1104.

[8] LiU, J. AND XU, G. (2012). Some asymptotic results of Gaussian random fields with varying mean functions and the associated processes. Ann. Statist. 40, 262-293.

[9] LiU, J. AND XU, G. (2014). On the conditional distributions and the efficient simulations of exponential integrals of Gaussian random fields. Ann. Appl. Prob. 24, 1691-1738.

[10] Liu, J., Zhou, X., Patra, R. And Weinan, E. (2011). Failure of random materials: a large deviation and computational study. In Proc. Winter Simulation Conf. 2011, IEEE, New York, pp. 3779-3789.

[11] Piterbarg, V. I. (1996). Asymptotic Methods in the Theory of Gaussian Processes and Fields. American Mathematical Society, Providence, RI.

[12] Taylor, J. And Adler, R. J. (2003). Euler characteristics for Gaussian fields on manifolds. Ann. Prob. 31, 533-563.

[13] Taylor, J., Takemura, A. And Adler, R. J. (2005). Validity of the expected Euler characteristic heuristic. Ann. Prob. 33, 1362-1396.

[14] Cirel'son, B. S., Ibragimov, I. A. and Sudakov, V. N. (1976). Norms of Gaussian sample functions. In Proceedings of the Third Japan-USSR Symposium on Probability Theory (Lecture Notes Math. 550), Springer, Berlin, pp. 20-41.

[15] URL: http://stat.columbia.edu/ jcliu/paper/OneDimDirichletDensity26FinalSupplement.pdf.

[16] URL: http://arxiv.org/abs/1309.3851. 